\title{
MPEG-SCORM: proposta de ontologia de metadados interoperáveis entre Televisão Digital e aplicações para o Ensino a Distância
}

\section{MPEG-SCORM: proposed metadata ontology for interoperable Digital Television and Distance Learning applications}

DOI: $10.46814 / 1 \mathrm{ajdv3n3-002}$

Recebimento dos originais: 05/04/2020

Aceitação para publicação: 05/05/2021

\section{Marcelo Correia Dos Santos}

Doutor em Engenharia pela FEEC UNICAMP

Universidade Estadual de Campinas, Faculdade de Engenharia Elétrica e de Computação, Campinas, SP

E-mail: marcelo.correia.santos@usp.br

\section{Yuzo Iano}

Professor Titular do Departamento de Telecomunicações da FEEC UNICAMP

Universidade Estadual de Campinas, Faculdade de Engenharia Elétrica e de Computação, Campinas, SP

E-mail: yuzo@unicamp.br

\section{RESUMO}

A convergência entre as mídias digitais propõe uma integração entre as TIC, focadas no domínio do multimídia (sob a responsabilidade do MPEG, Moving Picture Experts Group, constituindo o subcomitê ISO / IEC JTC1 SC29), e as TICE, (TIC para a Educação, geridas pelo subcomitê ISO / IEC JTC1 SC36), destacando-se os padrões MPEG, empregados na forma de conteúdo e metadados para o multimídia, e as TICE, aplicadas à Educação a Distância, ou e-Learning (o aprendizado eletrônico). Neste sentido, coloca-se a problemática de desenvolver uma correspondência interoperável de bases normativas, atingindo assim uma proposta inovadora na convergência entre as mídias digitais e as aplicações para e-Learning, essencialmente multimídia. Para este fim, propõe-se criar e aplicar uma ontologia de metadados interoperáveis para web, TV digital e extensões para dispositivos móveis, baseada na integração entre os padrões de metadados MPEG-21 e SCORM, empregando a linguagem XPath.

Palavras-Chave: Televisão Digital, e-Learning, Metadados, MPEG, SCORM, Convergência midiática.

\begin{abstract}
The convergence of digital media offers an integration of the ICT, focused on telecommunications and multimedia domain (under responsibility of the Moving Picture Experts Group, ISO/IEC JTC1 SC29), with the ICTE (the ICT for Education, managed by the ISO/IEC JTC1 SC36), highlighting the MPEG formats, featured as content and as description metadata potentially applied to the Multimedia or Digital TV and as a technology applied to e-Learning. Regarding this, it is presented the problem of developing an interoperable matching for normative bases, achieving an innovative proposal in the convergence between digital Telecommunications and applications for e-Learning, both essentially multimedia. To achieve this purpose, it is proposed to create an ontology for interoperability between educational applications in Digital TV environments and vice-versa, facilitating the creation of learning-metadata-based objects for Digital TV as well as providing multimedia video content as
\end{abstract}


learning objects for Distance Education. This ontology is designed as interoperable metadata for the Web, Digital TV and e-Learning, built on the integration between MPEG-21 and SCORM metadata standards, performing as a translator between the metadata standards between both domains, by employing the XPath language.

Keywords: Digital Television, e-Learning, Metadata, MPEG-21, SCORM, Convergence.

\section{TELEVISÃO DIGITAL E EDUCAÇÃO A DISTÂNCIA}

No atual contexto histórico, situamo-nos num momento de completude final da transição cujo movimento se dá a partir da tecnologia analógica em direção à digital em sua plenitude. Misturam-se antigos espectadores, hoje usuários interagentes, e multiplicam-se produtores de conteúdos digitais que oferecem conteúdos de áudio, vídeo, texto e imagem dotados de recursos interativos, em múltiplas mídias que convergem sobre a plataforma digital.

De maneira mais ampla, o advento da World Wide Web e o desenvolvimento do broadcasting digital, cloud computing e web semântica (BERNERS-LEE et al., 2001), colocaram em evidência novas fronteiras e novas possibilidades tecnológicas que permitiram não só a criação, mas possibilitaram a expansão do armazenamento e compartilhamento de recursos digitais em novos desdobramentos (PEREZ-SOLTERO et al., 2021).

Como uma Nova Tecnologia Digital da Informação e Comunicação, a Televisão Digital assume seu lugar como mídia expoente no processo de convergência digital, visto que, entre outros atributos, possibilita a coexistência de recursos hipermidiáticos convergentes.

No contexto brasileiro, a Televisão Digital, sob a ótica do SBTVD $^{1}$, foi concebida com o propósito de cumprir algumas missões como contrapartida à sociedade brasileira, entre elas levar inclusão digital e educação à população, em todas as regiões do país, vindo de encontro a uma demanda latente por inclusão e acesso à educação, mas também à produção e compartilhamento de conteúdo. A Educação a Distância é uma ferramenta importante para o cumprimento dessas metas, especialmente se associada à televisão, meio de comunicação presente em mais de $90 \%$ das residências brasileiras, demonstrando o valor social que a TV Digital pode prestar à população brasileira, em iniciativas como T-learning ${ }^{2}$, ou T-EaD ${ }^{3}$, assim como o valor econômico potencial que possui para conferir desenvolvimento ao país.

\footnotetext{
${ }^{1}$ A sigla SBTVD refere-se ao Sistema Brasileiro de Televisão Digital. Mais informações podem ser obtidas no site <http://sbtvd.cpqd.com.br>. Acesso em 10 fev. 2021.

${ }^{2}$ E-learning (aprendizado eletrônico) pela Televisão.

${ }^{3}$ Educação a Distância (EaD) via TV Digital.
} 
A investigação de Doutorado que vem sendo realizada no programa de Pós-graduação na área de Telecomunicações para Televisão Digital, na FEEC UNICAMP, insere-se nesse contexto ao concentrar-se na área de Tecnologias Digitais da Informação e Comunicação aplicadas à Educação (TICE), propondo um projeto de inovação tecnológica e de conhecimento por compreender a pesquisa de um novo padrão que integra as normalizações internacionais implementadas na área do multimídia $\left(\mathrm{SC}_{2}{ }^{4}\right)$, destacadamente a Televisão Digital Interativa, com as do domínio da Educação e suas tecnologias $\left(\mathrm{SC} 36^{5}\right)$.

Tecnicamente, a Televisão Digital faz parte do domínio do multimídia MPEG ${ }^{6}$ por utilizar como formato de exibição, atualmente, o padrão MPEG-4 AVC H264.

Entretanto, a padronização do universo do multimídia MPEG não se restringe ao MPEG-4, assim como não se restringiu ao já igualmente bem-sucedido MPEG-2. Há uma década e meia fazemse grandes projetos de pesquisa, experiências e implementações práticas em aplicações com excelente desempenho que se utilizam dos padrões MPEG-7 e MPEG-21. Além de outros consolidados, destacadamente o TV-Anytime ${ }^{7}$.

Após o MPEG-1 e 2, duas normas que tornaram possíveis o vídeo DVD e, mais recentemente, a Televisão Digital, o MPEG-4, 7 e 21 são as mais recentes normas na pauta do SC29. Estas três se articulam de forma interdependente entre si, prolongam-se ou se complementam de maneira coerente. Estas diferentes normas MPEG e suas características em termos de qualidade de encoding, ou codificação, de eficácia de compressão e de interatividade são sintetizadas na Figura 1:

\footnotetext{
${ }^{4}$ ISO/IEC JTC1 SC29: comitê que trata da encoding de som, de imagem e da informação multimídia e hipermídia.

${ }^{5}$ ISO/IEC JTC1 SC36: comitê que trata das tecnologias para a educação, a informação e a aprendizagem.

${ }^{6}$ Moving Picture Experts Group (MPEG), um grupo de trabalho da ISO/IEC estabelecido em 1988, com a missão de desenvolver padrões para representação codificada de dados relacionados em áudio e vídeo digital.

${ }^{7}$ Especificação de entrega de conteúdo multimídia e seus metadados para TV Digital. Disponível em: <http://www.tvanytime.org>. Acesso em 10 abr. 2021.
} 
Figura 1: Diferentes normas MPEG e suas características em termos de qualidade de encoding, de eficácia de compressão e de interatividade.

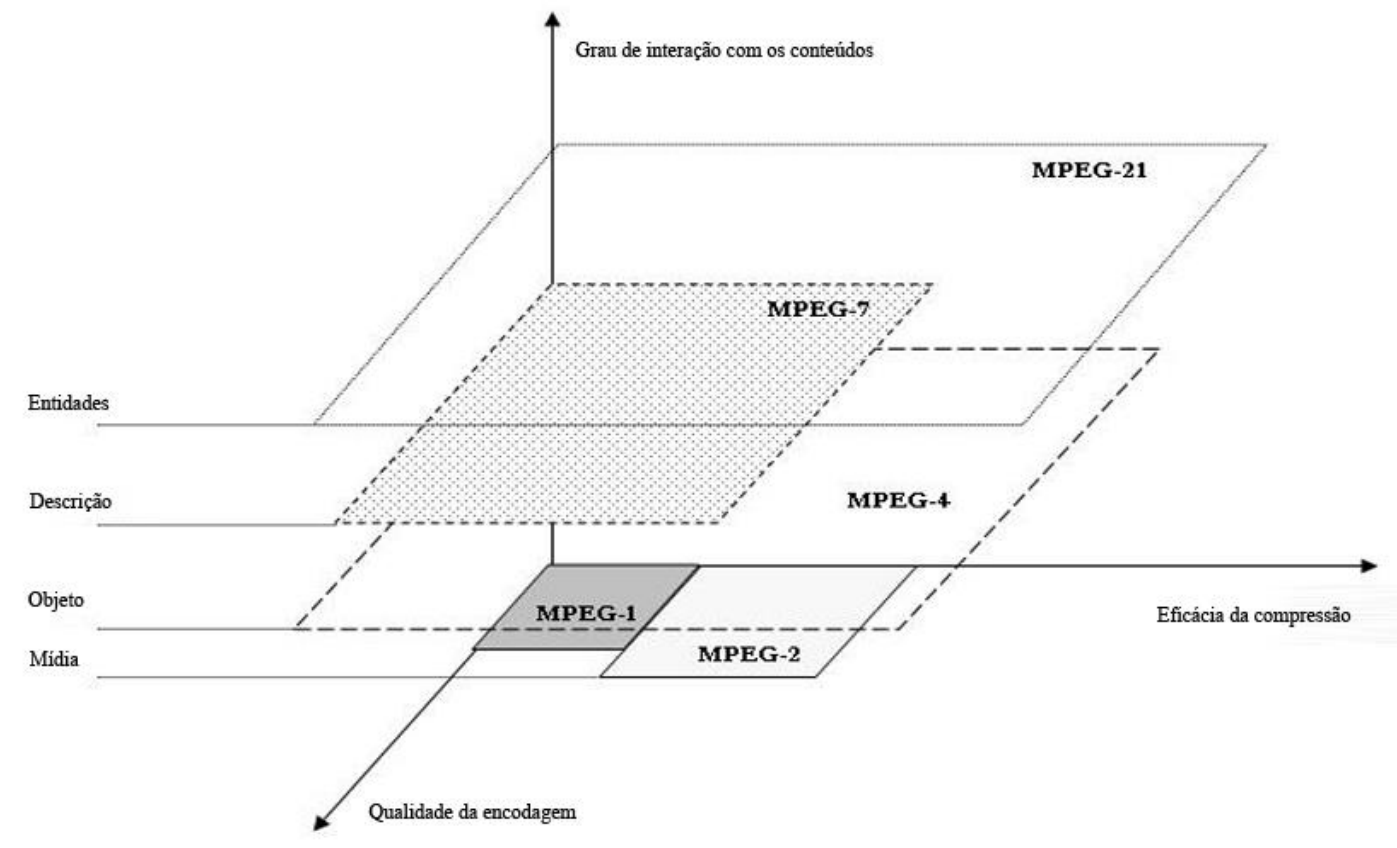

Fonte: LAQUET et al. (2009) - tradução livre.

Considerando os desafios de padronização das Tecnologias da Informação e Comunicação aplicadas à Educação, constitui-se um problema de engenharia de Televisão Digital ainda em desenvolvimento a questão de como a família MPEG Vídeo, por meio das normas MPEG-4, 7 e 21, poderia oferecer uma base normativa para a implementação de metadados associados às TICE.

Esse desenvolvimento necessita de uma instrumentalização de todos os componentes pedagógicos (além do próprio vídeo, imagem estática, som, texto, hiperlink etc) e deve se situar dentro de um quadro normativo a fim de garantir a interoperabilidade, reutilização e referenciamento pela maioria das plataformas ou ambientes virtuais de aprendizado, ou mesmo pela TV Digital ou outras mídias que venham a se localizar numa dimensão além desses ambientes clássicos educacionais.

A lógica referencial do corpus do documento digital é uma das premissas basais para os desenvolvedores de sistemas de informação para que seja possível criar uma normalização no tocante à engenharia de software (XML, MPEG-7, MPEG-21). Esses princípios são desenvolvidos em todas as dimensões pelo subcomitê que trata da padronização para as TICE, o SC36.

Entretanto, estando este estudo altamente implicado na normatização das TICE (SC36), constatou-se que a maior parte dos experts desta instância está não mais que vagamente consciente dos esforços de apropriação e de adaptação do MPEG-21 (e sem dúvida alguma também do MPEG-7 e 4) que devem ser feitos. Direitos autorais e copyright, assim como a gestão do $e$-jurídico, parecem fora do campo cultural da maioria dos experts do SC36. 
É bem menos o caso, por outro lado, dos delegados ou especialistas da $\mathrm{ADL}^{8}$, que desenvolveram o padrão SCORM, participam do SC36 e de certa forma propõem explorar o MPEG21 para solucionar uma família de funcionalidades normativas que se deve desenvolver para tornar as normas das TICE viáveis técnica e economicamente.

A ADL, desenvolvedora do padrão SCORM, intervém como parceiro nível A no SC36 atuando no subcomitê em questão, e sua estratégia é capitalizar sobre as normas de outros SC, e em particular procura atualmente fomentar a adoção da parte 5 do MPEG-21 (Rights Expression Language), para solucionar problemas de copyright, além de delegar às normas do LOM (ou outros formatos de metadados de aprendizagem como o Dublin Core ou o futuro MLR) o cuidado de descrever os recursos de aprendizagem em suas diferentes facetas. Claramente, esta descrição se tornaria concorrente das questões das normas multimídia da família MPEG.

\section{MPEG-4, 7 E 21 E METADADOS PARA TV DIGITAL E AS TICE}

Parece evidente a todo expert do SC36 que diversos segmentos deste subcomitê se encontram em processo de reformatação, uma vez que já estão resolvidos ou em curso de desenvolvimento normativo MPEG (Duta, Mitrea, Prêteux et al., 2008).

Em se apropriando e em adaptando as lógicas normativas MPEG (por meio do MPEG-4 e 7), torna-se possível desenvolver de forma muito mais focada a questão do vídeo interativo pedagógico propriamente dito. É contraproducente tender a resolver os problemas de gestão e integração do multimídia no âmbito do SC36, uma vez que são tratados por experts MPEG.

O MPEG-21 tornou-se a plataforma modular de desenvolvimento (o framework) e normalização, no sentido da integração global de todos os documentos multimídia. O multimídia não é produto de uma área do conhecimento específica, mas é uma consequência direta da normalização das práticas digitais, como os desenvolvedores de telefonia, de audiovisual, da informática, que se põe de acordo dentro das instâncias de normatização por lhes serem vantajosas a interoperabilidade e a compatibilidade, sob a égide do e-procurement ${ }^{9}$.

A normalização das TIC se situa mais e mais como uma prática consistindo em oferecer plataformas de produção, ou frameworks, à indústria. Apesar do MPEG-21 ter surgido de uma comunidade que se concentra sobre o áudio e o vídeo, o chamado MPEG-21 Framework pode acolher todo tipo de objetos digitais complexos, como o texto eletrônico, revistas digitais, dados científicos etc.

\footnotetext{
${ }^{8}$ Advanced Distributed Learning, iniciativa do governo americano datada de 1999, tendo como vetor o DoD (Departamento de Defesa), que tem a missão de recomendar e desenvolver padrões para software de treinamento.

${ }^{9}$ Compra e venda de suprimentos, trabalho e serviços através da Internet (seja B2B, B2C ou B2G), bem como outros sistemas de informação e de redes, tais como intercâmbio eletrônico de dados e planejamento de recursos empresariais.
} 
A força deste formado reside então na sua capacidade em oferecer uma plataforma geral padronizada para o sistema de produção e distribuição de todo conteúdo digital.

Como se pode constatar a partir da literatura científica (BORMANS, 2003; KALLI, 2004; PRÊTEUX, 2007) e da própria norma (ISO/IEC 21000-2:2003, 2003), o padrão MPEG-21 tem uma estrutura não rígida de metadados, e a Parte 2 da norma, DID, trata do Item Digital como a abordagem mais genérica para este propósito de uso de descrição estrutural dos metadados em objetos digitais de todo tipo.

A norma MPEG-21 comporta, hoje, 21 partes (MPEG ISO/IEC N5231, 2002). MPEG-21 é uma especificação de metadados baseada em XML que traz dois pilares fundamentais: a definição de uma unidade ou objeto fundamental de distribuição e transação, que é o Item Digital; e a noção de "leitor", a concepção dos usuários que interagem com o mesmo (MPEG-21, 2002).

O conceito central no âmbito do MPEG-21 é o de DI - Digital Item, ou Item Digital, definido na Parte 2 da norma (MPEG-21, 2003).

O DID, ou Digital Idem Declaration, é grosso modo um produto digital que pode ser simples ou composto. Um exemplo típico de um DI é aquele de uma página web, contendo diferentes recursos multimídia, como texto, imagem, vídeo, elementos de formatação (como folhas de estilo CSS), hiperlinks, scripts de programação dinâmica.

O MPEG-21 fornece os mecanismos descritivos de tais produtos digitais complexos. Em particular, as partes 2 (Digital Item Declaration) e 3 (Digital Item Identification) permitem, respectivamente, a especificação completa e estruturada dos DI; e a sua identificação e localização.

O uso do MPEG-21 DIDL (Digital Idem Declaration Language) como padrão genérico para a representação, catalogação e armazenamento de objetos digitais já foi proposto por BEKAERT et al. (2003).

O segundo conceito fundamental no formato MPEG-21 é o da descrição da produção e da interação com as mídias, por todos os envolvidos no processo, desde o produtor de conteúdo ao usuário final. Por isto, pode-se afirmar que o objetivo principal do MPEG-21 é definir as tecnologias necessárias para suportar o intercâmbio, acesso, consumo, comércio ou manipulação de Itens Digitais de forma eficiente e transparente (MPEG-21, 2002).

Deve-se destacar também a Parte 7 do padrão, DIA (Digital Item Adaptation), que padroniza os descritores e os esquemas de descrição, permitindo a adaptação dos conteúdos dos usuários, das redes, dos terminais ou ainda do ambiente de uso. Também a REL (Rights Expression Language) e a UD (User Description) tratam de descrever metadados que criem registro sobre essa relação entre produtor, conteúdo e usuários. 
O conjunto dos trabalhos do MPEG-21 situa-se em perfeita continuidade com aqueles realizados anteriormente no âmbito do MPEG-7. E muitos descritores do padrão MPEG-7 (2001a, 2001b) fazem parte do escopo do metadata schema do MPEG-21 (2002, 2003). Os descritores e esquemas de descrição correspondentes são desenvolvidos sob a responsabilidade do grupo MDS (Multimedia Desciption Schemes) e com a ajuda da mesma linguagem de descrição de dados fundada sobre a linguagem semântica de marcação XML.

A partir do padrão MPEG-21, KALLI et al. (2004) desenvolveram o conceito de Digital Broadcast Item Model (DBIM), que seria um padrão centrado em Digital Items com características específicas para o broadcasting da televisão digital.

Procurou, ainda, uma convergência entre os padrões DBIM (baseado no MPEG-21) e TVAnytime, criando um workflow integrado, na verdade, não uma integração de modelo de metadados na forma de uma ontologia.
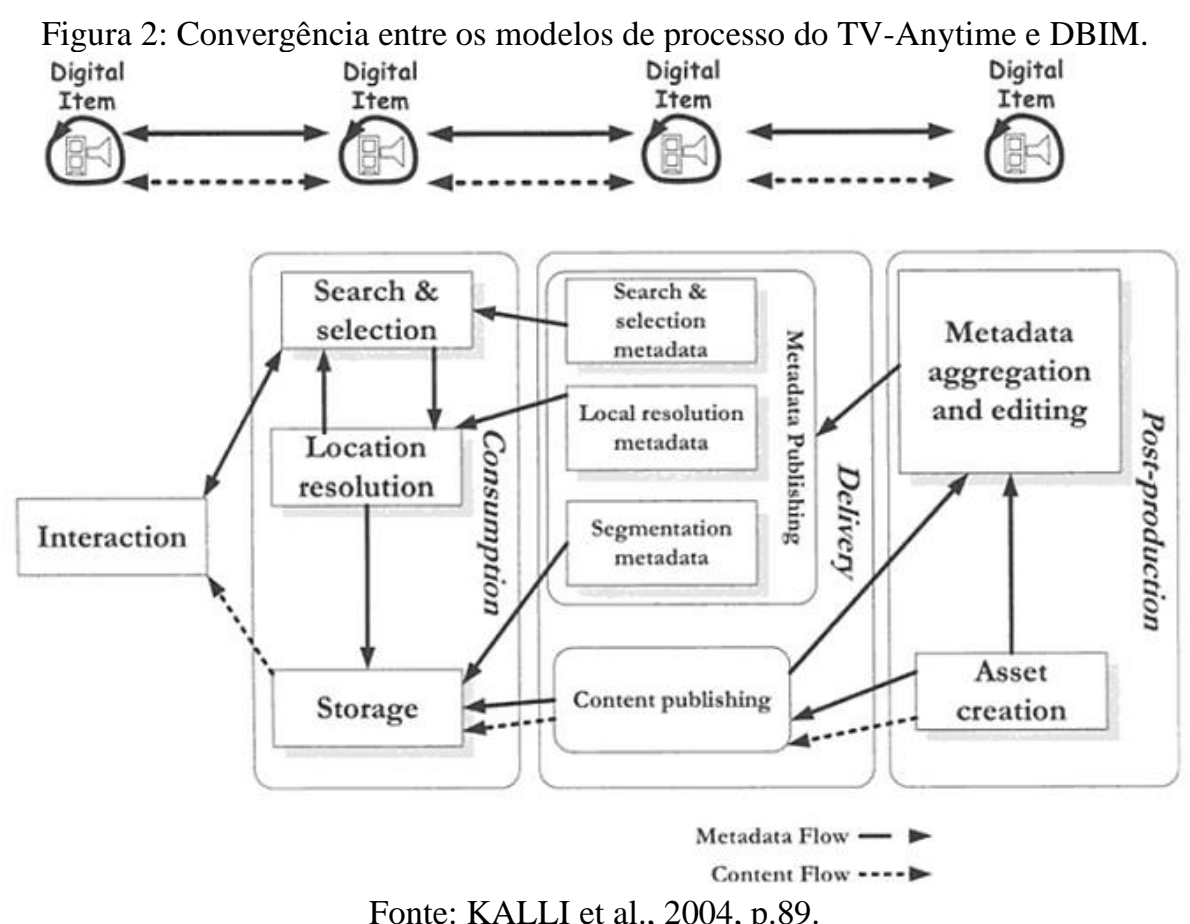

Em ALVES et al. (2006), LAQUET et al. (2009), HUNTER (2002), e GIGLIO et al. (2011 trabalho publicado nos Anais do Intercom), temos amostras de que a discussão envolvendo MPEG-7, MPEG-21 e TV-Anytime vem de longa data no universo da TV Digital, e mesmo a integração entre estes já foi discutida com propostas desenvolvidas (PFEIFFER, SRINIVASAN, 2000; CHRISTODOULAKIS et al., 2005). 


\section{OBJETO DE APRENDIZAGEM E METADADOS}

Qual a definição de Objeto de Aprendizagem (OA)? Segundo o padrão 1484.12.1 (Standard for Learning Object Metadata) do IEEE (IEEE LTSC, 2002, p.3), "um objeto de aprendizagem é definido como qualquer entidade, digital ou não digital, que pode ser utilizada para aprendizado, educação ou treinamento".

No universo digital, esta entidade pode, portanto, ser reutilizada ou referenciada durante o aprendizado apoiado por computador. O mesmo pode conter simples elementos como um texto ou um vídeo. Ou ainda, ele pode ser um hipertexto, um curso ou até mesmo uma animação com áudio e interação.

Segundo a IEEE LTSC (2002) o padrão LOM (Learning Object Metadata) focaliza o mínimo de atributos necessários para permitir que um Objeto de Aprendizagem seja gerenciado, localizado e avaliado. Os metadados permitem a catalogação e a codificação do objeto de Aprendizagem, tornandoo compreensível para as diversas plataformas.

SCORM não define um modelo de metadados: ele reconhece e recomenda enfaticamente o LOM como o padrão de facto (ADL, 2009, p. 4-65). Todavia, SCORM define o XML como sintaxe de representação dos metadados (XML binding).

\section{ONTOLOGIAS DE INTEGRAÇÃO ENTRE OS PADRÕES}

O MPEG-21, conforme já exposto, prevê a DIDL (Digital Item Declaration Languagem), porém também outros esquemas e suas linguagens, em outras das muitas partes da norma, para catalogação não só dos objetos, do fluxo da informação, como DII (Digital Item Identification), DIA (Digital Item Adaptation), mas também de direitos autorais (MPEG-21 Parte 5, REL - Rights Expression Language), CEL (Contract Expression Language), IPMP (Intellectual Property Management and Protection); e mesmo de casos de uso (UD - User Description).

Para se criar uma ontologia, ao se fazer um "match", uma correspondência e alinhamento entre os elementos do padrão SCORM com os do padrão MPEG-21 devem ser levadas em conta essas distintas linguagens e a importância de cada uma para o caráter extremamente abrangente e versátil do MPEG-21 como descritor de objetos, ou itens digitais.

A orientação dos trabalhos do JTC1 SC36, como se pode acompanhar pelo IEEE (BORMANS, BURNETT et al., 2003) baseia-se essencialmente sobre a portabilidade, a interoperabilidade e a adaptabilidade das tecnologias para a educação, o ensino e a aprendizagem. O SC36 não possui, portanto, vocação para duplicar ou mesmo estender os trabalhos previamente realizados por outros comitês técnicos, como o próprio SC29, o comitê do multimídia, que trata da codificação do som, imagem, da informação multimídia e hipermídia. 
Entretanto, o SC36 foi pioneiro em apontar a necessidade de sinergia do SCORM com o padrão MPEG-21, proposição todavia limitada ao tratamento de questões de direito autoral, copyright e, eventualmente, do e-commerce das TICE (Parte 5 da norma).

Em suma, as inter-relações possíveis entre padrões de metadados do MPEG e do e-Learning são esquematizadas na Figura 2:

Figura 3: Campos abrangidos pelos principais padrões das TICE.

\begin{tabular}{|c|c|c|c|c|c|c|c|c|c|c|c|c|c|}
\hline & 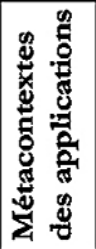 & 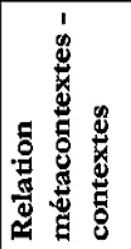 & 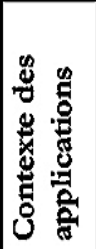 & 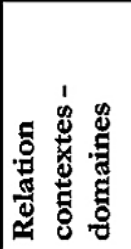 & 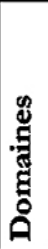 & 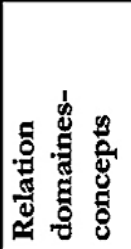 & 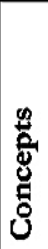 & 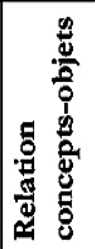 & $\frac{5}{\frac{2}{0}}$ & 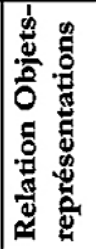 & 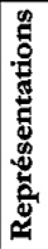 & 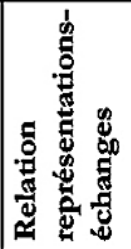 & 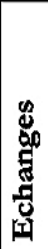 \\
\hline \multicolumn{14}{|c|}{ DUBLIN CORE } \\
\hline \multicolumn{14}{|l|}{ SCORM } \\
\hline \multicolumn{14}{|l|}{ LOM } \\
\hline \multicolumn{14}{|l|}{ MPEG-7 } \\
\hline MPEG-21 & & & & & & & & & & & & & \\
\hline
\end{tabular}

Fonte: BEN HENDA et al., 2008.

É possível realizar-se correspondências entre padrões de metadados, pois não são incompatíveis. Por exemplo, mapeando-se os metadados para e-learning DCMI e LOM, teremos a seguinte tabela de similaridade:

Tabela 1: Mapeamento entre metadados DCMI e SCORM.

\begin{tabular}{|l|l|}
\hline \multicolumn{2}{|c|}{ Correspondência entre Metadados DCMI / LOM } \\
\hline \multicolumn{1}{|c|}{ DCMI } & \multicolumn{1}{c|}{ LOM } \\
\hline DC.Identifier & General.Identifier.Entry \\
\hline DC.Title & General.Title \\
\hline DC.Language & General.Language \\
\hline DC.Description & General.Description \\
\hline DC.Subject & $\begin{array}{l}\text { General.Keyword ou Classification.Keyword com } \\
\text { Classification.Purpose igual a "Discipline" ou "Idea" }\end{array}$ \\
\hline DC.Coverage & General.Coverage \\
\hline DC.Type & Educational.LearningResourceType \\
\hline DC.Date & $\begin{array}{l}\text { LifeCycle.Contribute.Date com LifeCycle. } \\
\text { Contribute.Role igual a "publisher" }\end{array}$ \\
\hline DC.Creator & $\begin{array}{l}\text { LifeCycle.Contribute.Entity com LifeCycle. } \\
\text { Contribute.Role igual a "author" }\end{array}$ \\
\hline DC.OtherContributor & $\begin{array}{l}\text { LifeCycle.Contribute.Entity com o tipo de contribuição } \\
\text { especificado em LifeCycle. Contribute.Role }\end{array}$ \\
\hline DC.Publisher & $\begin{array}{l}\text { LifeCycle.Contribute.Entity com LifeCycle. } \\
\text { Contribute.Role igual a "publisher" }\end{array}$ \\
\hline DC.Format & Technical.Format \\
\hline DC.Rights & Rights.Description \\
\hline DC.Relation & Relation.Resource.Description \\
\hline DC.Source & Relation.Resource com Relation.Kind igual a "IsBasedOn" \\
\hline
\end{tabular}

Fonte: própria autoria. 
Conforme observa-se, estes identificadores vão desde os mais gerais, até os relativos a direitos autorais (o termo Rights, observável na penúltima linha da tabela), que corresponde como já abordado a um foco da ADL para integração de uma parte da norma MPEG-21 (Parte 5, relativa à linguagem de expressão de direitos, REL) ao padrão SCORM.

Para realizar-se a integração de padrões normalizados, a solução a ser implementada nesta pesquisa e se fazer um paralelo entre as categorias de metadados do SCORM e sua correspondência com os definidos pelos metadata schema do MPEG-21, e vice-versa. Seguindo a linguagem XPath, do W3C, como padrão para a formatação taxonômica dos termos, inicialmente foram mapeados os seguintes metadados do padrão SCORM, que fariam correspondência a um padrão do multimídia, como o MPEG-21, aderente à convergência de plataformas (como TV Digital, web, mobile).

Para coincidir com as especificações desses padrões de metadados o procedimento está mapeando suas categorias usando, nesta pesquisa, a linguagem XPath, pois foi feito o mapeamento e correspondência dos padrões de metadados DCMI - Dublin Core e SCORM, conforme mostrado na Tabela 1.

Como podemos observar na Tabela 2, esses identificadores abrangem desde os mais genéricos até os mais específicos, relacionados aos Direitos, por exemplo, que é foco da padronização ADL referente ao MPEG-21 e ao SCORM. O mapeamento é expresso em Sintaxe XPath.

Tabela 2: Mapeamento entre metadados DCMI e SCORM, especificação na linguagem XPath.

\begin{tabular}{|l|l|}
\hline \multicolumn{1}{|c|}{ SCORM } & \multicolumn{1}{|c|}{ Matching SCORM (LOM) / DCMI Metadata } \\
\hline /lom/general/identifier/entry & /dc/identifier \\
\hline /lom/general/title & /dc/title \\
\hline /lom/general/language & /dc/language \\
\hline /lom/general/description & /dc/description \\
\hline $\begin{array}{l}\text { /lom/general/keyword or } \\
\text { /lom/classification/keyword } \\
\text { com classification/purpose equals to "Discipline" or } \\
\text { "Idea" }\end{array}$ & /dc/subject \\
\hline /lom/general/coverage & /dc/coverage \\
\hline /lom/educational/learningresourcetype & /dc/type \\
\hline $\begin{array}{l}\text { /lom/lifecycle/contribute/date } \\
\text { com lifecycle/contribute/role equals to "Publisher" }\end{array}$ & /dc/date \\
\hline $\begin{array}{l}\text { /lom/lifecycle/contribute/entity } \\
\text { com lifecycle/contribute/role equals to "Author" }\end{array}$ & /dc/creator \\
\hline $\begin{array}{l}\text { /lom/lifecycle/contribute/entity } \\
\text { with the contributing type specified in } \\
\text { lifecycle/contribute/role }\end{array}$ & /dc/othercontributor \\
\hline $\begin{array}{l}\text { /lom/lifecycle/contribute/entity } \\
\text { com lifecycle/contribute/role equals to "Publisher" }\end{array}$ & /dc/publisher \\
\hline /lom/technical/format & /dc/rights \\
\hline /lom/rights/description & /dc/relation \\
\hline /lom/relation/resource/description & \\
\hline
\end{tabular}




\begin{tabular}{|l|l|}
\hline $\begin{array}{l}\text { /lom/relation/resource } \\
\text { com relation/kind equals to "IsBasedOn" }\end{array}$ & $/ \mathrm{dc} /$ source \\
\hline
\end{tabular}

Fonte: própria autoria.

A partir da Tabela 2, referente ao casamento entre os padrões de metadados SCORM e Dublin Core, é possível analisar uma projeção para os resultados esperados alcançados através do trabalho na ontologia híbrida posteriormente mapeada proposta, implicando por sua vez nos padrões de metadados SCORM e MPEG-21. Note-se a sintaxe da linguagem XPath utilizada para mapeamento e sistema de correspondência, de forma neutra, entre as duas estruturas de representação de metadados.

Foram constatadas coincidências entre os 15 (quinze) elementos apresentados no padrão Dublin Core e, por outro lado, exatamente 15 elementos equivalentes encontrados dentro da estrutura de metadados do SCORM, na verdade estrutura LOM, conforme anteriormente apresentado.

A Ontologia pretendida entre SCORM e MPEG-21 pode ser realizada combinando suas correspondências de sintaxe, através da linguagem W3C XPath.

O uso da linguagem XPath é direcionado para realizar o estudo comparativo de mapeamento entre os padrões de metadados SCORM e MPEG-21, aplicando esta metodologia. XPath é uma linguagem mantida pelo W3C com o objetivo principal de endereçar partes de um documento XML. Também é usada para testar se um código corresponde a um padrão ou a outro código.

Existem alguns recursos-chave em relação à linguagem XPath que foram fortemente considerados na decisão para esta metodologia proposta:

- XPath é uma sintaxe para definir partes de um documento XML

- XPath usa expressões de caminho para navegar em documentos XML

- XPath contém uma biblioteca de funções padrão

- XPath é um elemento importante em XSLT ${ }^{10}$

- XPath é uma recomendação W3C

O MPEG-21, conforme explanado, apresenta a DIDL (Digital Item Declaration Language), mas também outros esquemas e suas linguagens, dentro de outras das diversas partes da norma, para a catalogação de objetos e o fluxo de informações, caso também DII (Digital Item Identification) e DIA (Digital Item Adaptation); mas também para lidar com dados de direitos autorais (MPEG-21 Parte 5 REL - Linguagem de Expressão de Direitos), CEL (Linguagem de Expressão de Contrato), IPMP (Gerenciamento e Proteção de Propriedade Intelectual); e até mesmo casos de uso (UD - Descrição do Usuário).

${ }^{10}$ XSL significa EXtensible Stylesheet Language (e é aplicado a arquivos XML). 
A diretriz primordial dos trabalhos do JTC1 SC36, que pode ser acompanhada por artigos publicados pelo IEEE, baseia-se essencialmente nas premissas de portabilidade, interoperabilidade e adaptabilidade de tecnologias para educação, ensino e aprendizagem. O SC36 não pede, portanto, a ampliação dos trabalhos de outras comissões técnicas, como o próprio SC29, o comitê de mídia, que trata de codificação de som, imagem, multimídia e informação hipermídia.

Porém, o SC36 foi o pioneiro em apontar a necessidade de sinergia com o padrão SCORM MPEG-21, proposição esta, entretanto, limitada a abordar questões de copyright e eventualmente o ecommerce do ICTE (Parte 5 do padrão).

A normalização do MPEG-21 está em perfeita continuidade com as realizadas anteriormente na estrutura do MPEG-7. E muitos descritores padrão MPEG-7 fazem parte do escopo do esquema de metadados MPEG-21.

Os descritores e respectivos esquemas de descrição são desenvolvidos sob a responsabilidade do grupo MDS, cuja descrição dos dados está fundamentada na semântica da linguagem de marcação XML.

Para realizar a integração de padrões padrão, a solução que está sendo implementada é fazer a correspondência entre as categorias dos metadados SCORM e aquelas definidas pelo esquema de metadados MPEG-21.

Usando a linguagem XPath como ferramenta padrão para formatar as taxonomias e criar uma ontologia de convergência, primeiro foi mapeado o seguinte esquema de metadados SCORM CAM, que corresponderia ao esquema do padrão de multimídia MPEG-21, alinhado ao conceito de convergência da plataforma. Conforme representação na seguinte Tabela 3:

Tabela 3: Mapeamento entre metadados MPEG-21 DIDL e SCORM, especificação na linguagem XPath.

\begin{tabular}{|c|c|}
\hline Metadados SCORM & Metadados MPEG-21 \\
\hline /lom/general/identifier/entry & $\begin{array}{l}<\text { mpeg21> } \\
\text { /container/container@id }\end{array}$ \\
\hline /lom/general/identifier/catalog & $\begin{array}{l}<\mathrm{mpeg} 21> \\
\text { /container/descriptor/descriptor@id }\end{array}$ \\
\hline /lom/general/description & $\begin{array}{l}<\text { mpeg21> } \\
\text { /container/descriptor/statement }\end{array}$ \\
\hline /lom/general/title & $\begin{array}{l}<\operatorname{mpeg} 21> \\
\text { /item/descriptor/statement } \\
\text { Or } \\
\text { /item/descriptor/statement/foo:TITLE }\end{array}$ \\
\hline /lom/general/language & $\begin{array}{l}\text { <mpeg7> } \\
\text { /item/descriptor/statement/mpeg7/ } \\
\text { descriptionunit/creation/title@ @mlns:lang } \\
\text { Or } \\
<\text { mpeg21> } \\
\text { /item/descriptor/statement/rdf/lom:language }\end{array}$ \\
\hline /lom/general/keyword & $\begin{array}{l}<\text { mpeg21> } \\
\text { /item/descriptor/statement/rdf/lom:keyword }\end{array}$ \\
\hline
\end{tabular}




\begin{tabular}{|c|c|}
\hline /lom/general/coverage & $\begin{array}{l}<\text { mpeg21> } \\
\text { /item/descriptor/statement/rdf/lom:coverage }\end{array}$ \\
\hline $\begin{array}{l}\text { /lom/educational/ } \\
\text { learningresourcetype }\end{array}$ & $\begin{array}{l}<\mathrm{mpeg} 21> \\
\text { /item/descriptor/statement/ } \\
\text { rdf/lom:learningresourcetype }\end{array}$ \\
\hline /lom/lifecycle/contribute/role & $\begin{array}{l}<\text { mpeg7> } \\
\text { /item/descriptor/statement/mpeg7/creation/ } \\
\text { creator/role }\end{array}$ \\
\hline $\begin{array}{l}\text { /lom/lifecycle/contribute/ } \\
\text { date/dateTime }\end{array}$ & $\begin{array}{l}\text { <mpeg7> } \\
\text { /item/descriptor/statement/mpeg7/ } \\
\text { creationcoordinates/date/timepoint }\end{array}$ \\
\hline /lom/technical/format & $\begin{array}{l}<\mathrm{mpeg} 21> \\
\text { /item/component/resource@mimeType }\end{array}$ \\
\hline /lom/technical/location & $\begin{array}{l}<\text { mpeg21> } \\
\text { /item/descriptor/statement/rdf:description /lom:location }\end{array}$ \\
\hline /lom/technical/size & $\begin{array}{l}\text { <mpeg21> } \\
\text { /item/descriptor/statement/rdf:description/ } \\
\text { lom:size }\end{array}$ \\
\hline /lom/technical/duration & $\begin{array}{l}<\mathrm{mpeg} 21> \\
\text { /item/descriptor/statement/rdf:description/ } \\
\text { lom:duration }\end{array}$ \\
\hline $\begin{array}{l}\text { /lom/relation/resource/identifier/ } \\
\text { entry }\end{array}$ & $\begin{array}{l}<\text { mpeg } 21> \\
\text { item/component/resource@ @ref }\end{array}$ \\
\hline /lom/relation/resource/description & $\begin{array}{l}<\text { mpeg } 21> \\
\text { item/component/descriptor/statement }\end{array}$ \\
\hline /lom/rights/description & $\begin{array}{l}<\mathrm{mpeg} 7> \\
\text { /item/descriptor/statement/mpeg7/ } \\
\text { desciptionunit/copyrightstring }\end{array}$ \\
\hline
\end{tabular}

Fonte: própria autoria.

A Tabela 3 significa mapeamento e correspondência entre os dois padrões de metadados: SCORM e MPEG-21.

Os elementos mapeados de ambos os padrões devem ser combinados, com o objetivo de alcançar a ontologia híbrida que estabelece a convergência entre o padrão de metadados SCORM, do universo do e-Learning, com o padrão de metadados MPEG-21, do multimídia, padrão este propício ao propósito do projeto por ter como características ser interoperável e aplicável para a ampla gama de mídias digitais, incluindo a web, mídia móvel ou mesmo televisão digital.

\section{CONCLUSÕES}

O trabalho desenvolvido consistiu em justamente criar uma Ontologia relativa a essas taxonomias mapeadas, a fim de se propor, a partir de tal ontologia, uma integração entre os domínios do Multimídia (inclusa a Televisão Digital) MPEG e das TICE para EaD/e-Learning, ou seja: a convergência entre MPEG-21 e SCORM como um padrão para descrição de objetos utilizados para catalogação e para aplicação no aprendizado eletrônico, num sentido mais amplo, e no aprendizado eletrônico via Televisão Digital numa perspectiva específica do domínio da área de concentração desta pesquisa. 
Uma ontologia SCORM ${ }^{11}$ MPEG-21, fazendo-se a correspondência por meio da linguagem $X P a t h$, do $W 3 C$, já tem um desenvolvimento bem encaminhado, originando recentes artigos, produtos da pesquisa.

O emprego da linguagem XPath é direcionado à realização do estudo comparado de mapeamento entre os padrões de metadados SCORM e MPEG-21, aplicando-se esta metodologia. XPath é uma linguagem mantida pelo W3C com o objetivo primário de endereçar partes de um documento XML, também usada para testar se um código corresponde a um padrão, ou a outro código.

Foi possível obter resultados satisfatórios quanto às etapas superadas nesta pesquisa, no que diz respeito à implementação do objetivo específico de correspondência entre padrões de metadados dos domínios de conhecimento emitidos.

O trabalho de desenvolvimento consistiu na criação de uma Ontologia centrada nestas taxonomias mapeadas de forma a propor, a partir desta ontologia, uma integração entre as áreas da Multimídia (abrangendo a Televisão Digital), com o padrão MPEG-21, e das ICTE, que desenvolveu o padrão de objetos educacionais no formato de metadados SCORM, para o ensino à distância ou eLearning.

Ou seja, a convergência entre MPEG-21 e SCORM como padrão de descrição de objetos utilizados para catalogação e para uso em e-learning, e em um sentido mais amplo, o projeto de veiculação da modalidade e-learning via Televisão Digital, conferindo uma perspectiva específica do campo da pesquisa.

Contribui também para uma necessidade latente de integração entre os universos da Multimídia e do ICTE, representados pelos grupos de trabalho SC29 e subcomitês de normalização SC36, no âmbito da ISO / IEC JTC1, neste contexto contemporâneo de convergência digital pós-moderna.

\footnotetext{
${ }^{11}$ Sharable Content Object Reference Model, ou SCORM, o padrão de metadados para e-learning definido pela ADL e padrão de mercado global.
} 


\section{REFERÊNCIAS}

ADL Advanced Distributed Learning. SCORM 2004 4th Edition Content Aggregation Model (CAM), p.4-65, 2009. Disponível em: <http://www.moschorus.com/centre/MosPub/ documents/contenu/pages/SCORM_2004_4ED_CAM.pdf>. Acesso em 1 mai. 2021.

ALVES, L.G.P.; BRESSAN, G.; JUCÁ, P.; KULESZA, R.; SILVA, F. Análise comparativa de metadados em TV Digital. In: Workshop de Televisão Digital do SBRC2006, jun. 2006.

BEKAERT, J.; HOCHSTENBACH, P.; VAN DE SOMPEL, H. Using MPEG-21 DIDL to Represent Complex Digital Objects in the Los Alamos National Laboratory Digital Library. In: D-Lib Magazine, nov. 2003, vol. 9, n.11. <http://www.dlib.org/dlib/november03/bekaert/11bekaert.html>. Acesso em 10 abr. 2021.

BERNERS-LEE, T.; HENDLER, J.; LASSILA, O. The Semantic Web: a new form of web content that is meaningful to computers will unleash a revolution of new possibilities. In: Scientific American.com, 2001.

BORMANS, J.; BURNETT, I.; PEREIRA, F. VAN WALLE, R.; HILL, K.; MPEG-21: goals and achievements. In: IEEE Multimedia, out-dez 2003, vol.10, cap.4, p.60-70.

BEN HENDA, M.; PRETEUX, F.; VAUCELLE, A. et al. MPEG-21: une base normative pour le eprocurement des TICE. Évry (França): École d’Ingénierie Télécom SudParis, 2008.

CHRISTODOULAKIS, S.; KAZASIS, F.; POLYDOROS, P. et al. Ontology-based semantic indexing for MPEG-7 and TV-Anytime audiovisual content. In: Multimedia Tools and Applications, vol. 26, n. 3, p. 299-325, ago. 2005.

GIGLIO, K. et al. Metadados como viabilidade para organização e gerenciamento de conteúdo multimídia interativo no ambiente digital televisivo. In: Anais do XXXIV congresso brasileiro de ciências da comunicação - Intercom. Recife: 34, 2011. Disponível em: <http://www.intercom.org.br/papers/nacionais/2011/trabalhos.htm>. Acesso em 10 jan. 2021.

HUNTER, J. Reconciling MPEG-7 and MPEG-21 semantics through a Common event-aware metadata model. In: Cornell University Library. 2002. Disponível em: <http://arxiv.org/abs/cs/0210021v1〉. Acesso em 10 fev. 2021.

IEEE LTSC. IEEE Standard 1484.12.1 for Learning Object Metadata. New York: IEEE, 2002. ISO/IECJTC1/SC29/WG11/N5231. MPEG21 Overview v.5. 2002. Disponível em: <http://mpeg.chiariglione.org/standards/mpeg-21>. Acesso em 12 mar. 2021.

KALLI, S.; LUGMAYR, A.; NIIRANEN, S. Digital interactive TV and metadata: future broadcast multimedia. New York: Springer, 2004.

LAQUET, T.; PRÊTEUX, F.; VAUCELLE, A.; ZAHARIA, T. Les normes MPEG-7 et MPEG-21 pour la description des contenus multimédias. In: Conferência TAIMA'09, Hammamet (Tunísia), 2009.

LÓPEZ, M. R.; VILAS, A. F.; REDONDO, R. P. D.; ARIAS, J. J. P.; MUNÕZ, J. B. Adaptive learning objects for T-learning. In: IEEE Latin America Transactions, p.401-408, out. 2007. 
LYON, L.; PATEL, M.; CHRISTODOULAKIS, S. et al. Project n507618 DELOS: status report for work package 5. Joint Programme of Activities (JPA3), fev-set. 2006. Disponível em: <http://deloswp5.ukoln.ac.uk/project-outcomes/D5-0-2-report-draft/d5-0-2-report-draft.doc>. Acesso em 12 mar. 2021.

MPEG-21 ISO/IEC 21000-2:2003. Multimedia framework (MPEG21) Part 2: Digital Item Declaration. 2003. Disponível em: <http://www.iso.ch/iso/en/CatalogueDetailPage. CatalogueDetail?CSNUMBER=35366\&ICS1=35> . Acesso em 12 mar. 2021.

PEREZ-SOLTERO, A.; LOPEZ-MUÑOZ, M. E.; VALENZUELA, M. B. Tecnologías de la web semántica para el desarrollo de una memoria organizacional en la atención de usuarios en un departamento de tecnología. In: Latin American Journal of Development, v. 3, n. 2, p. 685-697, 3 mai 2021.

PFEIFFER, S.; SRINIVASAN, U. TV-Anytime as an application scenario for MPEG-7. In: Proc. ACM Multimedia, p. 89-92. Los Angeles (EUA), 2000.

VAUCELLE, A. MPEG-21: la norme des TICE du XXIème siècle? In: Rétrospective et Perspective H2PTM'09. Paris: Hermès-Lavoisier, 2009.

VICARI et al. Proposta de padrão de objetos de aprendizagem baseados em agentes (OBAA). In: LACLO Congresso Latinoamericano de Objetos de Aprendizagem. São Paulo, 2010. 